\title{
Anticonvulsant Hypersensitivity Syndrome
}

\section{Fittje}

\section{Citation}

M Fittje. Anticonvulsant Hypersensitivity Syndrome. The Internet Journal of Dermatology. 2000 Volume 1 Number 1.

DOI: $\underline{10.5580 / 298 \mathrm{e}}$

\begin{abstract}
Anticonvulsant Hypersensitivity Syndrome (AHS) is a delayed drug reaction associated with the use of carbamazepine, phenytoin, and phenobarbital. This uncommon, though potentially fatal, multisystem disorder appears to be a hypersensitivity reaction to the arene metabolites of these drugs. The most common clinical presentation of this disorder includes fever, rash and lymphadenopathy. Onset generally occurs within 2-4 weeks of initiating therapy, but may take as long as 3 months.
\end{abstract}

\section{DISCUSSION}

Carbamazepine, Phenytoin and Phenobarbital all share a common structural feature, an aromatic benzene ring. The normal in vivo behavior of this drug involves conversion of the aromatic benzene ring into a highly unstable arene oxide metabolite via the cytochrome $\mathrm{P} 450$ system. These metabolites are highly electrophilic compounds that disrupt cellular function. The most common theory behind the mechanism of AHS is believed to occur as a result of deficient epoxide hydrolase, an enzyme responsible for the detoxification of the arene oxide metabolite. A deficiency in this enzyme results in a buildup of the toxic metabolites, and the subsequent cytotoxicity and hypersensitivity ensues.

AHS is most common among elderly black males, and overall prevalence is higher in the African-American population. It occurs in about one in 1,000 to one in 10,000 exposures. It is postulated that there may be a genetic determining factor associated with AHS. Familial occurrence of hypersensitivity has been observed, and findings suggest that it follows an autosomal pattern of inheritance. While initial onset (with first drug exposure) may appear within one week to three months, symptoms may occur as soon as one day after re-initiating therapy with the same drug (rechallenge). Cross-sensitivity between these three anticonvulsants is very high. Between $50-75 \%$ of patients with AHS showed in vitro cross-sensitivity to the other two drugs. The cross-sensitivity between these three anticonvulsants may explain why patients worsen with subsequent use of the other two anticonvulsants associated with this syndrome. $1,{ }_{2}, 3$

\section{Figure 1}

CLINICAL FEATURES OF ANTICONVULSANT HYPERSENSITIVITY SYNDROME 4
FINDING INCEDENCE

Fever

Skin Rash

Lymphadenopathy

Hepatitis

Hematologic Abnormalities

(eosinophilia, atypical lymphocytosis)

Facial Edema

50

25

\section{SIGNS AND SYMPTOMS}

Over $60 \%$ of reactions involve more than one organ within the first 48 hours.1,2 Most patients present with a high fever ranging between 38 ( and 40(C, which may persist for several weeks. This fever is generally accompanied by a skin rash. The rash typically affects the upper trunk, extremities and face early on in the reaction, but can spread to the lower extremities. Often the palms and soles of the feet are spared. It can be described as an erythematous, edematous, papular rash, which may or may not be pruritic. Initial stages of the rash are patchy, later progressing into a more confluent pattern.

Another hallmark presentation of AHS is lymphadenopathy, 
a tender condition that can be localized or generalized. Most hyperplasia associated with lymphadenopathy is benign growth or resolves with discontinuation of the offending drug. Potentially fatal complications include hypersensitivity myocarditis and drug-induced hepatitis. 1,2,3 Patients who develop hepatitis (with jaundice) increase their chance for mortality up to $50 \% .3$

\section{TREATMENT}

The most important aspect of treatment is to withdraw the anticonvulsant immediately. Patients with AHS should not be treated with carbamazepine, phenytoin or phenobarbital. Alternative anticonvulsant therapy can be substituted in most cases with valproate, benzodiazepines, gabapenin, vigabtrin, and lamotrigine appropriate to the seizure disorder.

AHS can be progressive despite termination of therapy. To treat signs and symptoms of AHS, topical and oral corticosteroids (which may shorten the duration of the reaction), antihistamines, maintaining fluid and electrolyte balance, and wet wraps have all shown to have beneficial value.

\section{CONCLUSION}

Although rare, Anticonvulsant Hypersensitivity Syndrome can have potentially fatal consequences. This condition has been associated with the use of aromatic benzene anticonvulsants, including carbamazepine, phenytoin and phenobarbital. It occurs with varying onset, signs and symptoms, which may make diagnosis difficult. It is more prevalent among the African-American population, and may have a familial component associated with its occurrence.

\section{References}

1. Morkunas, Anthony R. PharmD, BCPS. Miller, Mary Beth, DO. Medical Toxicology "Anticonvulsant Hypersensitivity Syndrome.” Vol. 13;No. 4;Oct 1997;727-737.

2. Mylonakais, Elefthrios. Vittorio, Carmela C. Hollik, Deborah. Rounds, Sharon. The Annals of Pharmacotherapy

"Lamotrigine Overdose Presenting as Anticonvulsant Hypersensitivity Syndrome.” Vol. 33;May 1999;557-559. 3. Mahadeva, U. Al-Mrayat, M. Steer, K. Leen, E. Postgraduate Medical Journal "Fatal Phenytoin Hypersensitivity Syndrome.” Vol. 75;Dec. 1999;734-736. 4. Clinical Pediatrics, Vol.39;2/2000;111 


\section{Author Information}

Michele Fittje, Pharmacy Extern

Pharmacy Extern, Pharmacy, University of Colorado School of Pharmacy 